\title{
Heterochronous Suture Line Recurrences in the Jejunal Pouch following Total Gastrectomy for Stage II Gastric Cancer: A Case Report and Literature Review
}

\author{
Takamichi Yokoe a,d Michio Sato ${ }^{a, e}$ Masashi Yahagi ${ }^{a} \quad$ Murat Dogrua \\ Hiroto Fujisaki ${ }^{a}$ Masaharu Ogura ${ }^{a}$ Hiroshi Kawamata ${ }^{a}$ \\ Fumitaka Asahara ${ }^{a}$ Shin Takayama ${ }^{a}$ Hirohisa Harada ${ }^{a}$ Yoichi Tanaka ${ }^{b}$ \\ Jun Miyauchic, funichi Matsui ${ }^{a}$ \\ aDepartment of Surgery, Tokyo Dental College Ichikawa General Hospital, Ichikawa, \\ Japan; ${ }^{b}$ Division of Surgical Pathology, Clinical Laboratory, Tokyo Dental College Ichikawa \\ General Hospital, Ichikawa, Japan; 'Department of Pathology and Laboratory Medicine, \\ Tokyo Dental College Ichikawa General Hospital, Ichikawa, Japan; dDepartment of Surgery, \\ Keio University School of Medicine, Tokyo, Japan; ${ }^{e}$ Department of Surgery, International \\ Goodwill Hospital, Yokohama, Japan; 'Department of Central Laboratory, Saitama Municipal \\ Hospital, Saitama, Japan
}

\section{Keywords}

Gastric cancer · Suture line recurrence · Local recurrence

\begin{abstract}
We report the case of a 65-year-old male who developed heterochronous local recurrences of gastric cancer in the jejunal pouch (J-pouch) four times after total gastrectomy. He underwent total gastrectomy, J-pouch, and Roux-en-Y reconstruction for stage II gastric cancer in 2005. Four local recurrences appeared on the esophago-jejunal anastomosis, the suture line within the pouch, the esophago-jejunal anastomosis, and the anastomosis between the jejunum and Y-loop, which were resected by partial excision or endoscopic submucosal dissection. Suture line recurrence of gastric cancer is rare. The common features for each recurrence included the surgically negative resection margins, observation of the same histopathological subtype, absence of remote metastasis or peritoneal seeding, and the recurrence on the anastomotic suture line, suggesting that the cause of recurrence was the implantation of exfoliated cancer cells probably in the suture line. However, there is no established procedure for preventing implantation recurrence currently, the effectiveness of lumen lavage is suggested.
\end{abstract}


Yokoe et al.: Heterochronous Suture Line Recurrences of Gastric Cancer

\section{Introduction}

The reconstruction of a pouch reservoir as a gastric substitute has been advocated to provide better nutritional conditions and improve patients' quality of life after total gastrectomy for gastric malignancies [1]. Recurrences in the jejunal pouch (J-pouch) and its anastomotic vicinity are extremely rare. We report a case with heterochronous multiple recurrences in the J-pouch and anastomotic suture line after total gastrectomy for stage II gastric cancer.

\section{Case Report}

A 65-year-old asymptomatic male was diagnosed with gastric cancer (stage II) with a well-differentiated adenocarcinoma in the upper area of the stomach and underwent total gastrectomy, J-pouch, and Roux-en-Y (R-Y) reconstruction at another institution in 2005. Twenty-six months after the first operation, in 2007, the patient was diagnosed with a recur-
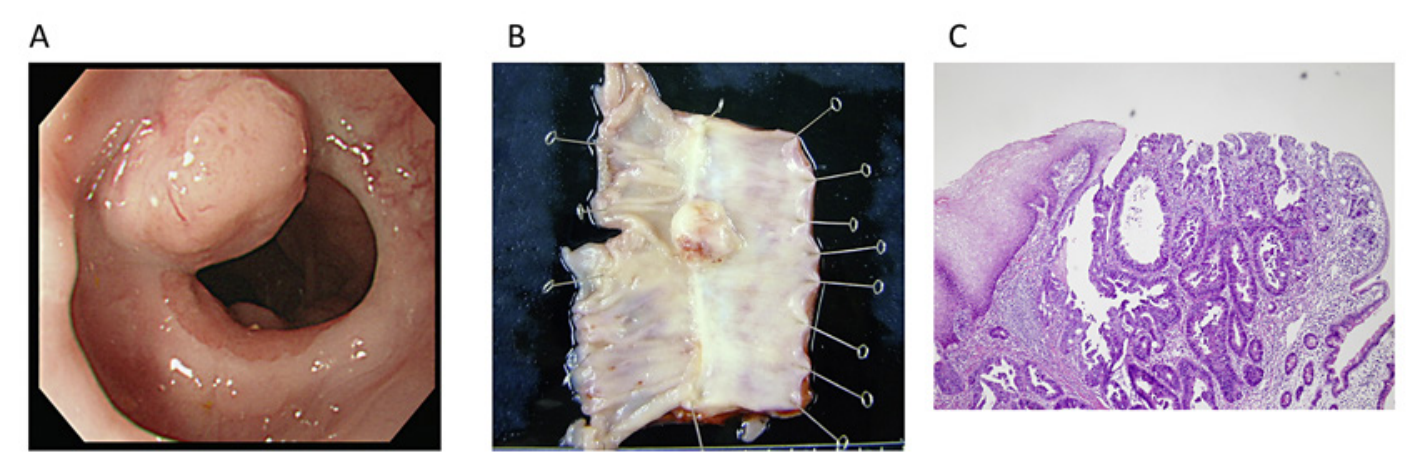

D

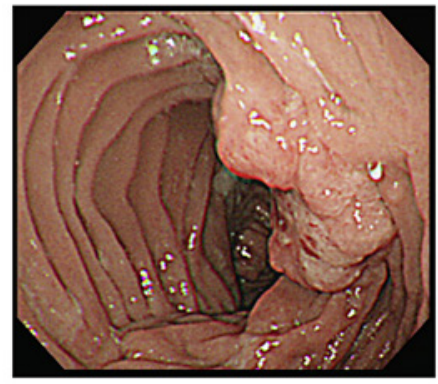

$\mathrm{E}$

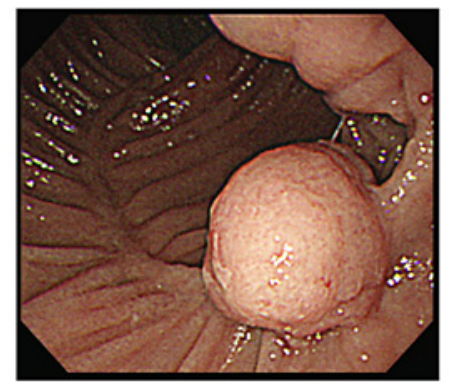

$\mathrm{F}$

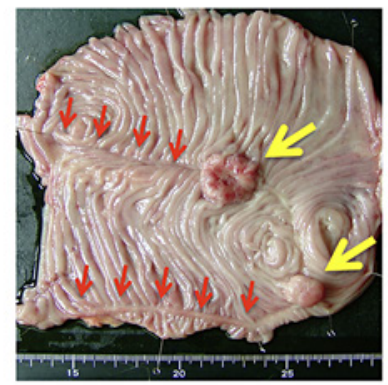

Fig. 1. First recurrence at the esophago-jejunal pouch anastomosis $(\mathbf{A}-\mathbf{C})$, and second recurrence at the anastomosis line within the J-pouch (D-F). A Macroscopic appearance of the irregularly elevated mass at the esophago-jejunal pouch anastomosis at the first recurrence $\mathbf{B}$ The trans-hiatal resection of the esophagojejunal anastomosis reveals a $2 \times 2 \mathrm{~cm}$ circular elevated lesion with irregular borders. $\mathbf{C}$ Histology of the tumor. A well-differentiated adenocarcinoma with focal papillary growth is present at the site of squamous and columnar epithelial junction. D, E Second recurrence at 2 sites of the anastomosis line within the J-pouch at 14 months after surgery. $\mathbf{F}$ The resection sample reveals a $2 \times 2 \mathrm{~cm}$ circular elevated lesion with irregular borders and a smaller $1 \times 1.5 \mathrm{~cm}$ elevated lesion. Both tumors are histologically well-differentiated adenocarcinomas with papillary structures, which resembles the previous recurrent tumor. Red arrows represent suture lines, yellow arrows represent recurrence tumors. 
rence at the esophago-jejunal pouch anastomosis and was referred to our institution. A gastrointestinal endoscopy revealed an irregularly elevated mass at the esophago-jejunal pouch anastomosis (Fig. $1 \mathrm{~A}$ and $2 \mathrm{~A}$ ), and the biopsy of the lesion confirmed an adenocarcinoma. The patient underwent the resection of the esophago-jejunal anastomosis using a trans-hiatal approach (Fig. 1B). The resected sample also revealed a circularly elevated lesion, measuring $2 \times 2 \mathrm{~cm}$, with irregular borders. Pathological examination of the resected specimen revealed a well-differentiated tubular adenocarcinoma with focal papillary structures (Fig. 1C). The margins of the specimen were negative for cancer, and there was no evidence of lymph node metastasis.

In 2008, 14 months after the second surgery, the patient was rediagnosed as having a tumor recurrence at 2 sites of the suture line within the J-pouch (Fig. 1D, E, and 2B) and he again underwent a J-pouch resection with a jejunal R-Y reconstruction. The resected sample contained a circularly elevated lesion, measuring $2 \times 2 \mathrm{~cm}$, with irregular borders and a

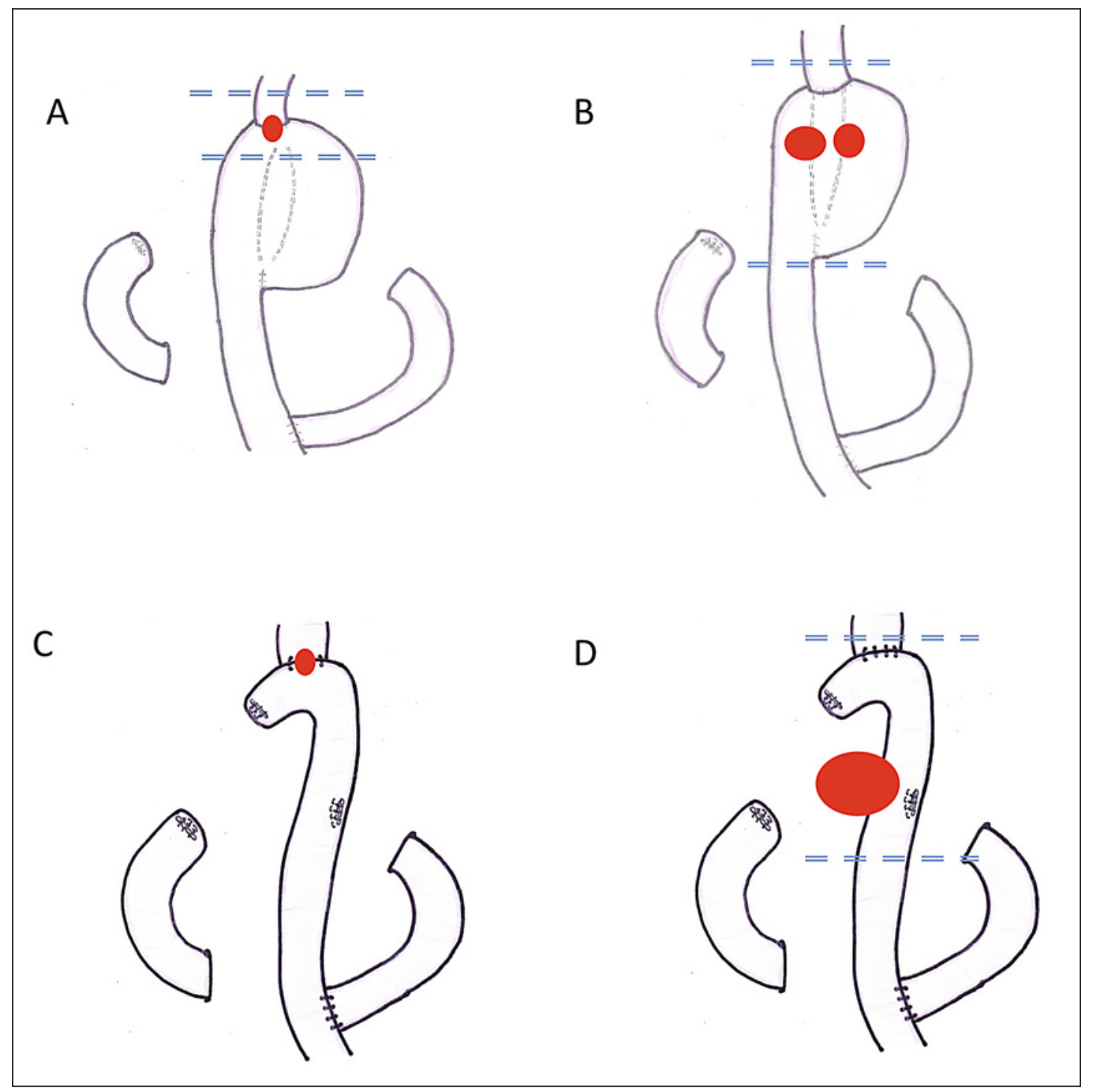

Fig. 2. Schematic diagram of the recurrence site. The site of recurrence is shown as red circles for the first $(\mathbf{A})$, second (B), third (C), and fourth (D) recurrence. 


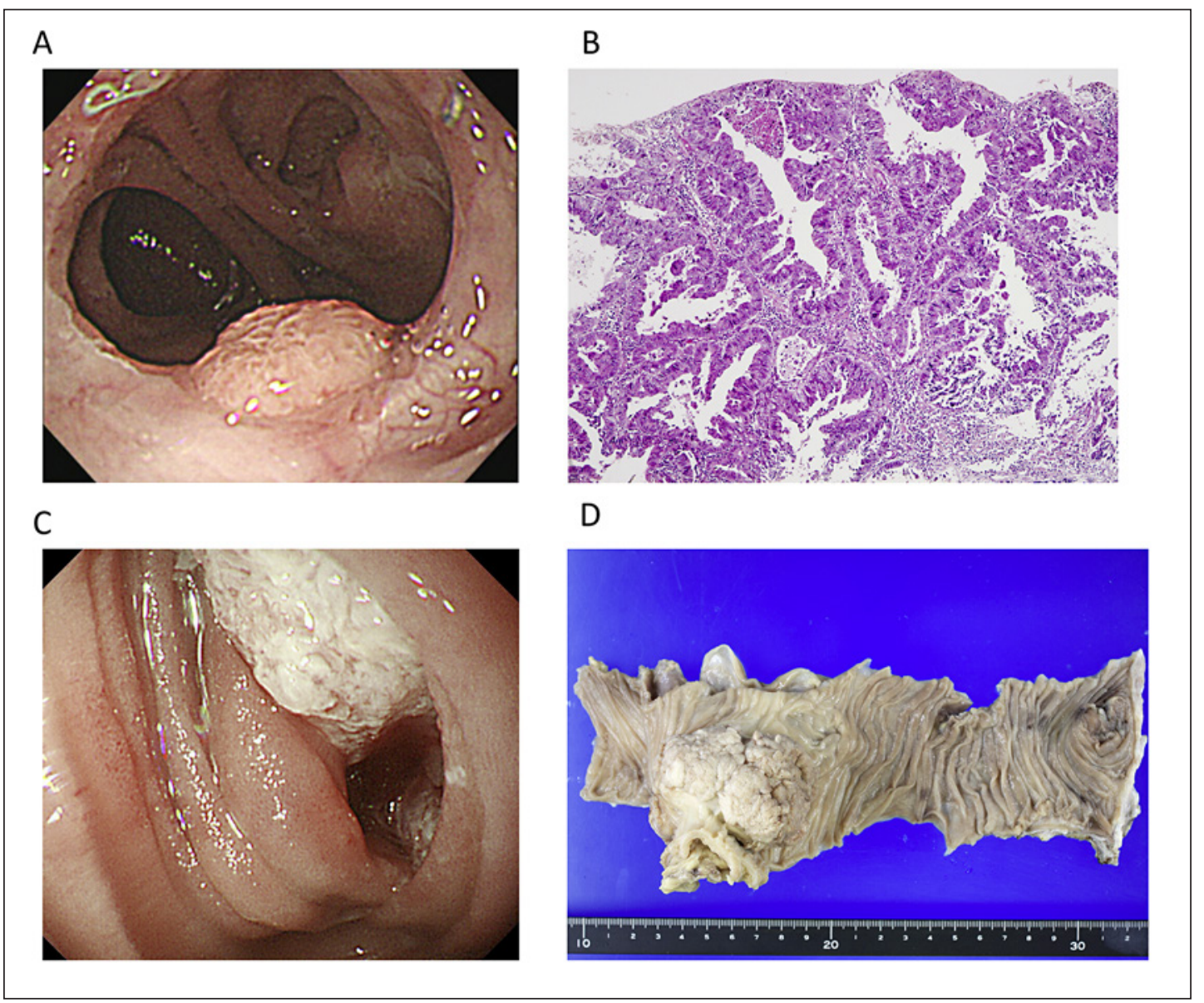

Fig. 3. Third recurrence at the esophago-jejunal anastomosis (A, B), and fourth recurrence in the close vicinity of the jejunum and Y loop (C, D). A The patient was diagnosed with a mucosal lesion at the posterior wall of the esophago-jejunal anastomosis for which the patient underwent endoscopic submucosal dissection. The resection sample reveals a $10 \times 5 \mathrm{~mm}$ circular elevated lesion with irregular borders. B The tumor is a histologically well-differentiated papillo-tubular adenocarcinoma. C An upper gastrointestinal endoscopy shows a fourth recurrence in the close vicinity of the jejunum and Y loop. D Gross pathology of the resection sample reveals a highly irregular cauliflower-like elevated mass measuring $5 \times 3 \mathrm{~cm}$ extending beyond the serosa. Histology of the tumor is a well-differentiated papillo-tubular adenocarcinoma.

smaller elevated lesion, measuring $1 \times 1.5 \mathrm{~cm}$ (Fig. 1F). The pathological examination of the specimen revealed a well-differentiated tubular adenocarcinoma with focal papillary growth in both lesions, findings similar to those of the previous surgical specimens. Again, the surgical margins were negative for cancer, and there was no evidence of lymph node metastasis.

Nine months after the third operation, a mucosal lesion at the posterior wall of the esophago-jejunal anastomosis was found in the patient (Fig. 2C and 3A), and he underwent endoscopic submucosal dissection. The pathological examination revealed a well-differentiated adenocarcinoma (Fig. 3B), but there were no tumor cells at the margins of the resected specimen. The patient was lost to follow-up but was re-admitted in 2012 with complaints of anorexia and gastric irritation 32 months after the endoscopic submucosal dissection. A gastrointestinal endoscopy revealed a tumor recurrence in the close vicinity of the anastomotic site between the jejunum and the Y-loop of the first operation, and computed tomography revealed tumor invasion into the transverse colon and lateral segment of the liver. The patient underwent a jejunal resection, partial resection of the liver and transverse and 


\section{Case Reports in \\ Oncology}

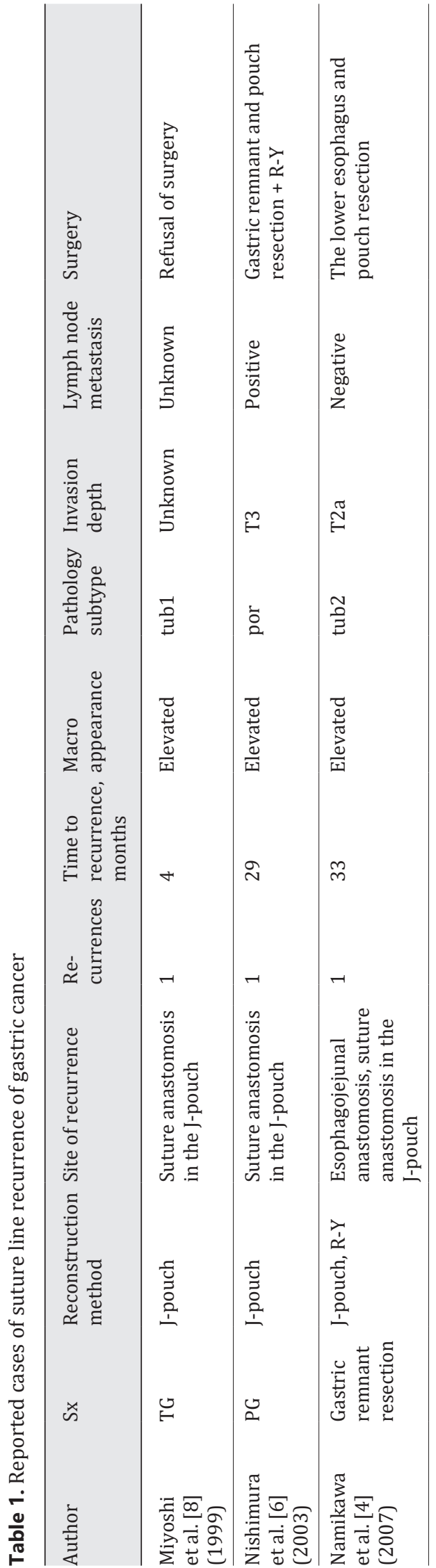

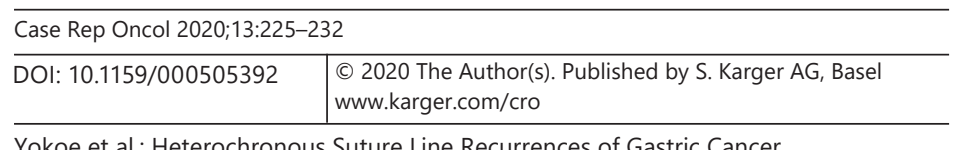

Yokoe et al.: Heterochronous Suture Line Recurrences of Gastric Cancer

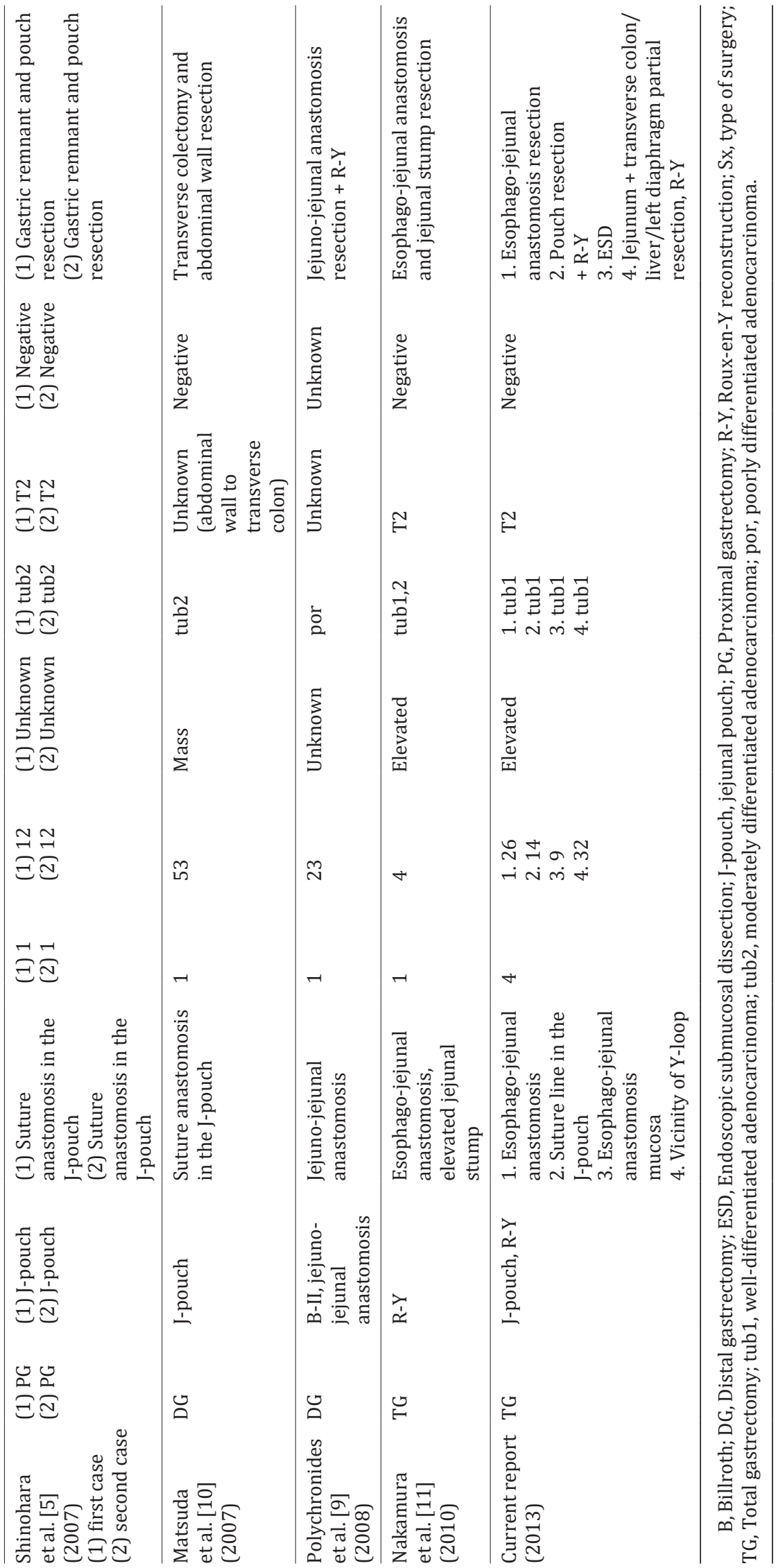


descending colons with a jejunal R-Y reconstruction (Fig. 2D and 3C). On gross examination of the resected sample, a highly irregular cauliflower-like elevated mass, measuring $5 \times 3 \mathrm{~cm}$, was found, which extended beyond the serosal surface (Fig. 3D). The tumor was histologically diagnosed as a well-differentiated adenocarcinoma forming papillary and ductal structures. The surgical margins of the resected specimen were free of tumor cells, and there was no lymph node metastasis. During follow-up, the patient showed no evidence of further recurrence and distant metastasis for one year after the last surgery, but eventually died of liver metastasis in 2015.

\section{Discussion}

The present case showed heterochronous local recurrences in the J-pouch following total gastrectomy for stage II gastric cancer. The formation of a pouch reservoir as a gastric substitute has been advocated to be clinically advantageous and to improve the quality of life after total gastrectomy for gastric cancer. Remnant gastric cancer after proximal or distal gastrectomy and recurrences following both types of gastrectomy are mostly in the stump [2]. The incidence of anastomotic or suture line recurrence after gastrectomy is reported to be $3-10 \%$ [3]. Suture line recurrence in the J-pouch is extremely rare.

The possible mechanisms of local stump recurrences after a total gastrectomy may be a submucosal or subserosal lymphatic spread, peritoneal dissemination, microinvasion of cancerous cells despite surgically negative resection margins, or possible implantation of exfoliated cancer cells. In our case, we suggest that lymphatic spread was unlikely since the tumor was a well-differentiated adenocarcinoma each time and neither lymph vessel invasion nor lymph node metastasis was seen in the specimens. The common features for each recurrence included the surgically negative resection margins, observation of the same histopathological subtype, absence of remote metastasis or peritoneal seeding, and the recurrence on the anastomotic suture line, suggesting that the cause of recurrence was the implantation of exfoliated cancer cells probably in the suture line.

We found seven reports of suture line recurrences after gastrectomy following a thorough PubMed and Medline research using the keywords "gastric cancer," "gastrectomy," "recurrence," "suture line," "jejunal pouch," and "anastomosis". The clinicopathological features of these cases, including those of our patient, are summarized in Table 1 [4-9]. We hereby report the nineth patient who developed J-pouch and anastomotic local recurrences four times after total gastrectomy for stage II gastric cancer. Several features can be identified from these cases. The depth of invasion was >T2; macroscopically, the tumor was of the elevated type; most cancers revealed a well-differentiated histological type; and the most common recurrence site was the suture line within the pouch. The period until recurrence was relatively long, with a median of 18.5 months.

There is no established method for preventing a suture recurrence of gastric cancer. Suture line recurrence is more common in colorectal cancers. Since it is thought that implantation occurs by the stapling device, due to the presence of cancer cells in the anastomosis portion, lavage of the rectum before anastomosis is a standard process. In gastric cancer cases, it has been reported that the positive washing cytology rate significantly decreases due to inside lumen lavage before esophageal dissection in total gastrectomy. Lavage before anastomosis may possibly prevent suture recurrence. 


\section{Conclusion}

Suture line recurrence of gastric cancer is rare, and this is the first case to present four heterochronous recurrences. Suture line recurrence by the implantation of exfoliated cancer cells features a greater depth of invasion, an elevated macroscopic type tumor, a well-differentiated type tumor, and the suture line within the pouch as the recurrence site, with a relatively long period until relapse. Although currently there is no established procedure that could prevent implantation recurrence, the effectiveness of lumen lavage is suggested.

\section{Statement of Ethics}

The authors have no ethical conflicts to disclose. The patient has given informed consent to publish their case including publication of images.

\section{Disclosure Statement}

The authors have no conflicts of interest to declare.

\section{Funding Sources}

No financial support was received for this submission. The authors have no financial or proprietary interest in any material or method mentioned.

\section{Author Contributions}

T.Y., M.S., and M.Y. interacted with the patient. T.Y., M.S., M.Y., and M.D. identified and acquired relevant reports. Y.T. and J.M. performed pathological diagnosis of the tumors. T.Y. drafted the report. All authors critically reviewed the report. All authors read and approved the final submitted version.

\section{References}

1 Gertler R, Rosenberg R, Feith M, Schuster T, Friess H. Pouch vs. no pouch following total gastrectomy: metaanalysis and systematic review. Am J Gastroenterol. 2009;104(11):2838-51.

2 Sugano MTM, Kajiyama Y, Hashimoto T, Nobukawa B, Hayashida Y. A case of successfully resected stomal recurrence of gastric cancer developed 13 years after total gastrectomy (in Japanese). Nihon Rinsyo Geka Gakkai Zasshi (J Jpn Surg Assoc). 2004;65:2913-7.

3 Papachristou DN, Karas M, Fortner JG. Anastomotic recurrence in the oesophagus complicating gastrectomy for adenocarcinoma of the stomach. Br J Surg. 1979;66(9):609-12.

4 Namikawa T, Kobayashi M, Okamoto K, Okabayashi T, Akimori T, Sugimoto T, et al. Recurrence of gastric cancer in the jejunal pouch after completion gastrectomy. Gastric Cancer. 2007;10(4):256-9.

5 Shinohara T, Kashiwagi H, Nakada K, Nimura H, Mitsumori N, Ohmura Y, et al. Suture line recurrence in the jejunal pouch after curative proximal gastrectomy for gastric cancer: report of two cases. Hepatogastroenterology. 2007;54(78):1902-4.

6 Nishimura M, Honda I, Watanabe S, Nagata M, Souda H, Miyazaki M. Recurrence in jejunal pouch after proximal gastrectomy for early upper gastric cancer. Gastric Cancer. 2003;6(3):197-201.

7 Miyoshi K, Fuchimoto S, Ohsaki T, Sakata T, Ohtsuka S, Takakura N. Long-term effects of jejunal pouch added to Roux-en-Y reconstruction after total gastrectomy. Gastric Cancer. 2001;4(3):156-61. 
8 Miyoshi K, Fuchimoto S, Ohsaki T, Sakata T, Takeda I, Takahashi K, et al. Suture line recurrence in jejunal pouch replaced after total gastrectomy for gastric cancer. Gastric Cancer. 1999;2(3):194-7.

9 Polychronidis A, Laftsidis P, Giatromanolaki A, Perente S, Bounovas A, Simopoulos C. Suture-line recurrence at a jejunojejunal anastomosis after gastrectomy for gastric cancer. Gastric Cancer. 2008;11(1):59-63.

10 Matsuda S, Yonei A, Kawano F, Taneda Y, Ichinari H, Mine K. Case report of a patient with a gastric cancer recurrence induced by implantation (in Japanese). Nihon Rinsho Geka Gakkai Zasshi (J Jpn Surg Assoc). 2007;62(2):279-81.

11 Nakamura H, Hiramatsu K, Katoh T, Suzuki M, Shibata Y, Yoshihara M, et al. A case of suture line recurrence in the esophagojejunostomy and the elevated jejunal stump after total gastrectomy for gastric cancer (in Japanese). Nihon Shokaki Geka Gakkai Zasshi (Jpn J Gastroenterol Surg). 2010;43:1117-22. 